\title{
LEARNERS' INTERPRETATIONS OF RECASTS
}

\author{
Helen Carpenter \\ Georgetown University
}

\author{
K. Seon Jeon \\ Columbus State University
}

David MacGregor and Alison Mackey

Georgetown University

\begin{abstract}
A number of interaction researchers have claimed that recasts might be ambiguous to learners; that is, instead of perceiving recasts as containing corrective feedback, learners might see them simply as literal or semantic repetitions without any corrective element (Long, in press; Lyster \& Ranta, 1997). This study investigates learners' interpretations of recasts in interaction. Videotapes of task-based interactions including recasts and repetitions were shown to advanced English as a second language students $(N=34)$. Although both groups viewed the teacher's feedback (recasts, repetitions, or other), one group saw video clips that had been edited to remove the learners' nontargetlike utterances that had triggered the feedback, and another group saw the same video clips with the initial nontargetlike utterances included. After each clip, learners in both groups were asked to indicate whether they thought they were hearing a recast, a repetition, or something else. A subset of learners $(n=14)$ provided verbal reports while they evaluated the clips. Results show that learners who did not overhear initial learner utterances were significantly less successful at distinguishing recasts from repetitions. The verbal protocol data suggest that learners were not looking for nonverbal
\end{abstract}

We are grateful to Bo Ram Suh for her help with data collection and coding and Rebecca Sachs for her help with editing. We would also like to thank Mohammed Louguit from the Center for Applied Linguistics for statistical assistance. We are grateful for the comments made by the anonymous SSLA reviewers who helped us to improve the paper. Despite the assistance of these individuals, any errors remain our own.

Address correspondence to: Helen Carpenter, Department of Linguistics, ICC 460, Georgetown University, Washington, D.C., 20057; e-mail: carpenth@georgetown.edu. 
cues from the speakers. A post hoc analysis suggests that morphosyntactic recasts were less accurately recognized than phonological or lexical recasts in this study. These findings suggest that the contrast between a problematic utterance and a recast contributes to learners' interpretations of recasts as corrective.

Long's (1996) interaction hypothesis stated that the linguistic modifications made as a result of breakdowns in communication between second language (L2) learners and their interlocutors might be especially helpful in the development of the learner's L2 system. The negotiation for meaning and recasts that can accompany communication breakdowns are assumed to provide learners with negative feedback and positive input related to their problematic production. This feedback can facilitate development in part because it comes at a moment when the gap between the learner's system and the target language is apparent to the learner. Long defined a recast as a discourse move that "rephrase $[\mathrm{s}] \mathrm{a}[\mathrm{n}] \ldots$ utterance by changing one or more sentence components (subject, verb, or object) while still referring to its central meanings" (p. 434). Many recent studies have found that recasts can be effective in promoting L2 development, supporting the claims of the interaction hypothesis (see, for example, summaries in Gass, 2003; Long, in press; Mackey, in press).

Nonetheless, some researchers have questioned the accessibility of the negative feedback available in recasts on the grounds that recasts can be ambiguous to the learner. Because recasts often occur in the same discourse contexts as noncorrective repetitions and other sorts of feedback without explicit cues that identify them as corrective in nature, it has been suggested that recasts might be interpreted as alternative ways of expressing the same meaning (Allwright, 1975; Fanselow, 1977; Lyster, 1998b; Lyster \& Ranta, 1997; Panova \& Lyster, 2002). In other words, the nature of the interaction may obscure the negative feedback provided in recasts and learners may fail to see the difference between their erroneous utterances and the corrections supplied by their interlocutors in recasts. For example, based on a study conducted with young L2 learners in a French immersion program, Lyster (1998a) argued that recasts are potentially ambiguous, especially in a classroom setting, where the primary focus is on meaning. In his words,

Recasts may be less successful at drawing learners' attention to their nontarget output-at least in content-based classrooms where recasts risk being perceived by young learners as alternative or identical forms fulfilling discourse functions other than corrective ones.... Thus, recasts of grammatical errors probably do not provide young classroom learners with negative evidence, in that they fail to convey what is unacceptable in the L2. (p. 207)

Selective attention to negative feedback plays an important role in the interaction hypothesis, where it is claimed to be a mediating factor in linguistic 
development (Gass, 2003; Long, 1996; Mackey, in press). However, if learners do not selectively attend to and recognize the negative feedback contained in recasts, then the contribution of recasts to learning might be attributed to the positive evidence that they contain or to the enhanced salience of the positive evidence, as Leeman (2003) suggested. Even though discussions in the SLA literature regarding whether and how learners perceive the feedback contained in recasts have been helpful in raising concerns and indicating directions for research, the investigation of these empirical questions requires more data-based studies. A better understanding of how learners interpret recasts and other kinds of utterances, such as repetitions, is an important and necessary step in describing the relationship between interactional processes and L2 learning. By developing new methodological approaches to address these questions, this paper aims to drive interaction research forward while adding to the research base in this area. To contextualize the study, we now turn to a review of learners' interpretations of recasts in the literature on recasts and L2 development, followed by a discussion of recasts as complex discourse structures.

\section{RECASTS, AMBIGUITY, AND L2 DEVELOPMENT}

Recasts have come to play a central role in claims about interaction-driven L2 development. Over the past 10 years, empirical research has suggested that recasts can facilitate (short-term) L2 learning; evidence for their positive impact comes from an increasing range of studies conducted in both experimental and classroom contexts (Ayoun, 2001; Braidi, 2002; Doughty \& Varela, 1998; Han, 2002; Havranek, 2002; Iwashita, 2003; Leeman, 2003; Long, Inagaki, \& Ortega, 1998; Mackey \& Philp, 1998; Muranoi, 2000; Oliver, 1995, 2000; Philp, 2003). Building on this research, the emphasis has expanded from investigating whether recasts are useful for learning to how recasts impact L2 development. For example, some have wondered how learners distinguish recasts from noncorrective repetitions, if they do so at all (Lyster, 1998a, 1998b; Lyster \& Ranta, 1997; Panova \& Lyster, 2002). Others have explored the modulatory effects of cognitive-developmental (Mackey, 1999; Mackey, Philp, Egi, Fujii, \& Tatsumi, 2002; Philp), linguistic (Han; Mackey, Gass, \& McDonough, 2000; Philp), and contextual (Iwashita; Oliver \& Mackey, 2003; Pica, 2002; Sheen, 2004) factors in the recognition of recasts. However, relatively little is known about the components of recasts or about exactly how they lead to language development. Thus, claims about the cognitive processes activated by recasts remain somewhat speculative. This is in part because recasts are complex discourse structures; they provide both implicit negative feedback and positive evidence. Moreover, some researchers also argue that because recasts are provided immediately after a nontargetlike form, the salience of the positive evidence is enhanced (Leeman, 2003, in press; Saxton, 1997). Next, we will first review some of the findings on recasts and 
L2 development in relation to the issue of ambiguity and then turn to a discussion of their status in discourse

Learners' perceptions about recasts have been studied by Philp (2003), who examined the extent to which nonnative speakers (NNSs) notice native speakers' (NSs') reformulations of their interlanguage grammar through recasts in dyadic interaction. In her experimental study, 33 adult English as a second language (ESL) learners participated in oral communication tasks in NS-NNS dyads and received recasts of their nontargetlike question forms. Attention to recasts was assessed through accurate immediate recall. Philp reported an overall high rate of noticing of recasts and found that three variables constrained accurate recall: the developmental level of the learner, the length of the recast, and the number of changes in the recast. From this, she concluded that the attentional resources and processing biases of learners modulated the extent to which they noticed the gap between their nontargetlike utterances and the corrections found in recasts.

Reporting on a small-scale laboratory study, Han (2002) hypothesized that because recasts are complex, they should be more beneficial for linguistic forms that are already in the process of being internalized than for forms that are unfamiliar to learners. She suggested that the frequency and saliency of the input in recasts were possible factors that influence their effectiveness in promoting morphosyntactic development and argued that intensive recasting in laboratory conditions aids development in morphosyntax by making linguistic features more noticeable in the input.

Another discussion of frequency and saliency comes from Iwashita (2003), who also reported positive outcomes for recasts in a study that investigated the role of task-based interaction in the learning of two Japanese morphosyntactic targets (locative constructions and te-form verbs). She found that NS interactional moves that contained only positive evidence about the two target structures were more frequent during task-based language learning than those that contained implicit negative feedback, including recasts. However, recasts were found to be more beneficial for short-term L2 development. Based on these results, Iwashita claimed that recasts, although not as frequent as simple models, might be much more salient than NS interactional moves that contain positive feedback, thus leading to significant gains in L2 development.

However, descriptive studies of content-based communicative classrooms in French immersion programs in Canada (e.g., Lyster, 1998a, 1998b; Lyster \& Ranta, 1997; Panova \& Lyster, 2002) have led Lyster and colleagues to cast doubt on the effectiveness of recasts in fostering L2 development in classroom contexts. After examining the discourse contexts in which recasts appeared, Lyster (1998b) claimed that recasts do not lead to student-generated repair, despite being the most frequently occurring feedback type in L2 classrooms. As mentioned earlier, Lyster (1998b) attributed the lack of modified output to the potential ambiguity of recasts, and he pointed out that they occur in the same discourse context as simple repetitions and claimed that the corrective element in recasting is often perceived by learners as confir- 
mation of meaning. In more recent work, Lyster (2004) compared the effects of recasts and prompts on young L2 learners' rule-based systems, which have been argued to be sensitive to feedback following form-focused instruction (Skehan, 1998). His quasi-experimental study was conducted in French immersion classrooms and used four production tasks (two written and two oral) to assess the students' abilities to assign grammatical gender in French. In terms of feedback type, recasts were shown to be less effective than prompts in leading to improvements, especially on the written production tasks. Lyster (2004) argued that learners who are prompted to use more targetlike forms are more likely to retrieve these forms in subsequent language processing than learners who simply hear recasts of these forms. Lyster (2004) related this finding to the ambiguity of recasts and potential difficulties involved in noticing morphosyntactic errors, and he concluded that recasts might not be the most effective type of feedback to use in communicatively oriented classrooms in comparison to other feedback types such as prompts.

It must be noted, however, that Lyster's (1998a, 1998b, 2004) results were obtained in a specific immersion setting and have yet to be replicated in other settings. In response to claims that the research setting might influence the effectiveness of recasts in promoting linguistic development, several recent studies have examined the possible role of interactional context-also with respect to ambiguity and uptake-as a factor that affects the learning opportunities provided (Oliver \& Mackey, 2003; Pica, 2002; Sheen, 2004). For example, Pica conducted a study in a subject-matter content-based classroom in which she examined student-teacher interactions in order to determine the extent to which learners and their teachers modify their interactions as they take part in activities related to subject-matter content. The data collection consisted of videotaping and audiotaping two classes (film and literature) in a university-level ESL program. Pica's analysis indicated that teachers did not directly address the majority of students' nontargetlike utterances. In fact, a large portion of the discourse was characterized by a paucity of interactional features considered helpful for L2 learning, such as negotiation of meaning, negative feedback (recasts), and pushed output. Pica attributed this to the nature of the communicative tasks; the discussion activities in these classes were mostly open-ended and they rarely required comprehensibility and accuracy of subject content.

In a classroom study of child L2 learners, Oliver and Mackey (2003) investigated the opportunities for, provision of, and use of feedback with young ESL learners (6-12 years old). Based on the teachers' responses to learner utterances, the researchers broke down the interactional context into four categories: content, management, communication, and explicit language. They then used stimulated recall protocols to ascertain that their coding of the data matched the teachers' perceptions. Results showed that opportunities for feedback, as well as its provision and use, vary depending on the interactional context of the exchange. For example, they found that teachers provided recasts most often-and learners used them most often-in explicit language- 
focused contexts. They also showed that $85 \%$ of the recasts were followed by modified output in these sorts of discourse contexts, whereas recasts never resulted in modified output in classroom management-focused contexts. This analysis suggests that results such as Lyster's (1998b) might be profitably subjected to additional analysis of the classroom discourse based on context.

This point is well illustrated in a study by Sheen (2004), who used Lyster and Ranta's (1997) coding scheme to compare teachers' corrective feedback and learners' uptake across a range of different communicative classroom contexts. Sheen adopted Lyster and Ranta's definition of uptake: "a student's utterance that immediately follows the teacher's feedback and that constitutes a reaction in some way to the teacher's intention to draw attention to some aspect of the student's initial utterance" (p. 49). Her analysis of the databases reported in Lyster and Ranta (French immersion), Panova and Lyster (2002; Canadian ESL), and Loewen (2002; New Zealand ESL), as well as original data collected in Korean English as a foreign language (EFL) contexts, confirmed that recasts were the most frequent feedback type used by teachers in communicative language classrooms. Sheen also found that although all of these instructional settings were communicatively oriented, the rates for uptake differed according to the context. Both uptake and repair (operationalized as uptake that leads to a correction of the error treated by the teacher) following recasts were greater in the New Zealand ESL and Korean EFL contexts than in the French immersion and Canadian ESL contexts, and Sheen argued that something about the nature of the teachers' recasts might have contributed to this contextual difference-a point we return to later. However, the use of uptake as an outcome measure may be a red herring (Mackey \& Philp, 1998). Sometimes, learners respond to the recasts by modifying their output, but not always. Regardless, recasts have been shown to be beneficial (Ayoun, 2001; Braidi, 2002; Doughty \& Varela, 1998; Han, 2002; Havranek, 2002; Iwashita, 2003; Leeman, 2003; Long et al., 1998; Mackey \& Philp; Muranoi, 2000; Oliver, 1995, 2000; Philp, 2003).

Mackey et al. (2000) also explored the possibility that learners' perceptions about recasts provided during task-based dyadic interaction might vary depending on the focus of the feedback. Learners' perceptions about recasts were collected using stimulated recall protocols with two groups of L2 learners (learners of ESL and Italian as a foreign language). The results showed that the ESL learners often recognized the feedback on lexis and phonology, but they did not often recognize morphosyntactic feedback as such. The learners of Italian recognized only $24 \%$ of the morphosyntactic feedback and also tended to perceive this kind of feedback as being about lexis. Lexical feedback episodes, on the other hand, were correctly perceived about two thirds of the time (66\%). Based on these results, Mackey et al. argued that both the nature and the target of the feedback might affect the accuracy of learners' perceptions.

In the most comprehensive overview of recasts in language learning to date, Long (in press) argued that the utility of recasts in SLA should not be rejected 
simply because of the possibility of ambiguity in some settings, such as immersion classrooms. Long also observed that Lyster's (1998b, 2004) skepticism about the developmental utility of recasts is based on immediate uptake, which cannot automatically be equated with learning. As Oliver (1995) first argued in her study of negative feedback in the conversation of child NS-NNS dyads, there is often no opportunity for immediate uptake (also known as learner repair or modified output) following recasts. Mackey et al. (2000) also pointed out that even if learners sometimes fail to identify feedback as such, this cannot be taken to mean that the feedback is not beneficial for language development. Mackey and Philp (1998) showed an association between recasts and learning independent of learners' immediate responses. Furthermore, a multiple regression analysis by Iwashita (2003) showed that recasts had a greater impact on short-term morphosyntactic development than other interactional moves, such as models. Citing results by Gass and Varonis (1994), Mackey (1999), and Ohta (2000), Long (in press) suggested that recasts might have delayed effects in language development and that the efficacy of recasts should not be discounted due to the absence of an overt oral response.

To sum up, although some researchers have drawn attention to the potential ambiguity of recasts and the questionable accessibility of the corrective element contained in them, a good deal of empirical research has shown that recasts can facilitate L2 learning. It has been argued that their utility should not be denied simply because they are not followed by uptake in certain contexts. Important next steps are to identify what makes recasts effective, when, and for whom, as well as to identify whether recasts of certain kinds and of certain forms are more effective than others. These are complex questions, but some pieces of the picture are emerging. For example, research has shown that opportunities for feedback and uptake might vary according to context (Loewen, 2002; Sheen, 2004) and that learners' perceptions might be influenced by the linguistic target of the feedback (Mackey et al., 2000). The ability to recall a recast has been linked to the length and number of changes in the recast (Philp, 2003). The developmental level of the learner in relation to the linguistic target of the recast has also been shown to be important, as have attentional resources, claims about working memory, and processing biases (Han, 2002; Mackey, 1999; Philp). Havranek's (2002) classroom research also suggested that the type of recast and response influences learning outcomes. Frequency and saliency also appear to be important factors (Han; Iwashita, 2003; Leeman, 2003), and it has been suggested that learners might be able to distinguish recasts from other discourse moves on the basis of paralinguistic and extralinguistic cues (Farrar, 1992; Long, in press; Lyster, 1998b; Sheen). Taken together, these findings point to the importance of isolating the various features of recasts in an attempt to understand which factors enable learners to recognize their corrective nature. Recasts are complex discourse structures, and the salience of the linguistic information contained in themwhether it serves as positive evidence or corrective feedback for learnersmust be investigated with respect to their unique discourse context. These 
ideas have been explored in both first language (L1) and L2 acquisition research, as will be discussed in the following subsection.

\section{Recasts as Complex Discourse Structures}

Using a constrained definition of recasts as reformulated utterances that correct learner errors, it is possible to propose a mechanism through which the negative feedback contained in recasts is made salient. Saxton's (1997) contrast theory of negative input states that "the unique discourse structure created in the juxtaposition of child error and adult correct form can reveal to the child the contrast, or conflict, between the two forms, and hence provide a basis for rejecting the erroneous form" (p. 139). Thus, Saxton and others in the L1 acquisition literature (see, for example, the summary in Nicholas, Lightbown, \& Spada, 2001) have proposed that a child's recognition of feedback-as in a recast-can act as a springboard for perceiving the adult form as a correct alternative to the child form. In a discussion of nonrecast responses, Saxton further noted, "it is assumed that other kinds of reformulation the adult might produce will not be misinterpreted by the child as being relevant to the correction of grammatical errors" (p. 146). Applied to SLA, Saxton's hypothesis predicts that learners can disambiguate recasts from other types of utterance if they understand, based on the immediate context of the utterance, that recasts contain negative feedback.

As mentioned previously, however, the complex nature of recasts makes it difficult to understand the source of their developmental benefits. For this reason, Leeman (2003) experimentally isolated the components of recasts to explore the separate effects of negative evidence, positive evidence, and enhanced salience of positive evidence. Spanish noun-adjective agreement morphology was selected as the linguistic target because, according to Leeman, it is realized via bound unstressed morphemes and, thus, has limited communicative value and low perceptual salience. Because the only two groups to show significant gains were the recasts group and the group exposed to positive evidence with enhanced salience (and no negative evidence), Leeman suggested that the benefits of recasts can be explained by enhanced salience of positive evidence. So, although recasts have usually been classified as implicit negative evidence, they also provide positive evidence with enhanced salience of target forms, and this positive evidence has been associated with learning. The nature of recasts as complex discourse structures has drawn researchers' attention to how and what learners perceive when presented with recasts.

In summary, the fact that recasts are complex and potentially ambiguous is recognized and discussed throughout the literature. Research has demonstrated that interactional context and setting can affect the quantity and quality of recasts as well as the production of modified output and L2 learning outcomes. Further investigations of recasts are clearly warranted, and one 
important unresolved issue concerns how learners recognize the corrective nature of recasts when they so often co-occur in contexts in which simple repetitions or confirmations are also provided.

\title{
Interpreting Recasts
}

Several researchers have discussed how learners distinguish recasts from repetitions. In the L1 acquisition literature, Farrar (1992) noted:

\begin{abstract}
What specific cues children are using in corrective recasts to correct their sentences is not clear at this point. There are a number of potential cues as to the corrective aspect of these recasts, such as the contrast between the adjacent sentence pairs or the intonation of the recast. These cues may be differentially associated with these recasts and contribute to children's elimination of certain incorrect rules. (p. 95)
\end{abstract}

In the SLA literature, Long (in press) suggested that extralinguistic cues might help disambiguate recasts from other types of feedback: "There is some suggestive evidence that it is subtle prosodic and extra-linguistic cues, such as facial expressions, which help with the disambiguation (Haig, 1995), although the fact that written recasts have also been found effective (Choi, 2000; Ayoun, 2001) means that verbal and immediate visual cues cannot be essential" (pp. 24-25). Additionally, Lyster's (1998b) data indicated that learners' reactions to recasts were different from their reactions to repetitions, which may suggest that the learners interpreted the two discourse moves differently. Lyster argued that when learners are able to distinguish recasts from repetitions, they do so on the basis of nonlinguistic cues provided by their teacher. Finally, as mentioned earlier, research by Sheen (2004) suggested that recasts provided in at least some contexts (New Zealand ESL and Korean EFL) were characterized by rising intonation or emphasis, often accompanied by stress. However, direct measures of learners' noticing of these recasts were not carried out in these studies.

Given these claims, it seems particularly important to investigate both the immediate discourse context and the presence of other cues as possible variables that mediate learners' recognition of the corrective nature of recasts. To investigate the ambiguity issue with recasts, the research questions addressed in the current study were as follows: (a) Do learners recognize the corrective nature of recasts when the recasts are removed from their immediate discourse context? (b) Do learners identify any nonlinguistic cues when deciding whether utterances are recasts or repetitions? These questions were investigated using a methodology unique to interaction research. The contribution of this methodology will be discussed at the end of this paper. We now turn to the details of the current study. 


\section{METHOD}

The description of methodology used in this study is discussed in the following subsections: (a) operationalizations, (b) materials creation, (c) participants, (d) procedure, and (e) coding.

\section{Operationalizations}

Recasts were operationalized as the NS's reformulation of all or part of a problematic learner utterance that corrected the error(s) without changing the central meaning of the utterance (cf. Long, 1996; Lyster \& Ranta, 1997; Mackey \& Philp, 1998). Recasts are always corrective in nature; that is, they are contingent on learners' errors (Long) and they may be embedded within declarative or interrogative utterances. They may be isolated, providing no additional meaning, or they may be incorporated into longer utterances in which additional meaning is provided (Lyster, 1998b).

Repetitions were operationalized as NS utterances that followed and repeated all or part of a learners' targetlike utterance (cf. Lyster \& Ranta, 1997). Like recasts, repetitions may be realized as declarative or interrogative utterances. They may be isolated (repetitions of part or all of the NNS utterances without additional information provided) or they may be incorporated into longer utterances that supply additional meaning (Lyster, 1998b). The examples in (1) and (2), taken from data collected for the current study, illustrate an initial problematic utterance (indicated by an asterisk) followed by a recast (1), and a targetlike utterance followed by a repetition (2). ${ }^{1}$

(1) Recast

NNS: *Huge wave crashed.

NS: A huge wave crashed.

(2) Repetition

NNS: He enjoyed fishing.

NS: Okay, he enjoyed fishing.

These definitions of recasts and repetitions were used in creating the stimulus videotapes.

\section{Materials Creation}

A videotape stimulus was used in this study to provide participants with authentic recasts and repetitions. To create the stimulus, 26 adult learners from an English as a second language program in a medium-sized urban university were videotaped as they carried out a communicative task-based activity in dyads with one of two NSs who were also researchers in the present 
study. Each dyad performed the tasks separately in a research room where a video camera was set up. The interlocutors were seated across from each other at a table. The learners, ranging in age from 18 to 39 years, were enrolled in high-intermediate- or advanced-level classes and came from a variety of L1 backgrounds. Two researchers-English NSs aged 30 and 36 years, one female and one male-led the activity. Both held master's degrees in teaching ESL and had taught ESL or EFL professionally for 5 and 10 years, respectively, prior to commencing research. Each session of stimulus creation took approximately $60 \mathrm{~min}$. Initially, learners participated in an instructional activity (10 min), followed by an interaction activity (20 min). Upon completion of these activities, learners provided comments on the interaction activity through stimulated recall sessions $(30 \mathrm{~min})$ that are not discussed in the current article.

The lesson, which pertained to ocean waves, provided exposure to novel vocabulary and conditional structures. The initial instructional component was based on a chapter in a content-based ESL textbook that focused on English and geography and was judged by the researchers to contain a sufficient amount of unfamiliar vocabulary for high-intermediate and advanced learners (Kessler, Lee, McCloskey, Quinn, \& Stack, 1996). The NSs provided new vocabulary to the learners about technical aspects of waves (e.g., crest, trough) and used conditional structures to describe various geophysical phenomena related to the formation and movement of waves. NNSs were not required to speak during the initial instructional phase, although numerous clarification checkpoints were built into the instructional component. ${ }^{2}$ In any discussions that arose, the NSs were instructed to provide recasts and repetitions where appropriate and otherwise to respond as they normally would (i.e., they were permitted to improvise). However, to ensure that the input provided to learners was consistent in terms of form and content, both between NS interlocutors and across participants, the lesson was scripted in advance and both NSs practiced beforehand to avoid overreliance on the script during instruction. The script was developed by the researchers based on their prior experience as ESL/EFL teachers. A set of illustrations provided visual support for conceptual information related to the subject matter of the lesson (e.g., how waves are formed in the open ocean).

Following the instructional phase, the NS interlocutors and the NNSs engaged in picture-based task activities that provided practice discussing waves and using factual and counterfactual conditional forms to make causeand-effect statements. There were three tasks of increasing complexity (Robinson, 2001), each designed to provide opportunities for the learners to produce the targeted vocabulary and forms. In this way, learners could potentially produce lexical, phonological, and morphosyntactic errors that could be recast by the NSs. For the interaction activities, only the instructions were scripted in advance; all discussion was spontaneous. The NSs were trained and instructed to provide recasts, repetitions, and other naturally occurring discourse moves as they deemed appropriate. Descriptions of the three tasks 
appear in the appendix. Together, the lesson and tasks were designed to encourage learners to use language associated with describing cause-andeffect relationships in English while applying their knowledge of concepts discussed in the lesson. Thus, the activities were content-focused and designed to provide practice in discussing the information presented during instruction.

As noted, these dyadic task-based interactions between the learners and the NSs were videotaped. The camera was focused on the NSs in order to capture any gestures or other nonverbal behaviors that accompanied their interactions, and a microphone captured their recasts, repetitions, and other discourse moves. To create the stimulus videotapes for the present study, three of the researchers coded the full dataset with respect to the discourse functions of the NSs' utterances, and a master template of video clips of each of the NS interlocutors was created, using only instances with $100 \%$ intercoder reliability. From this coded dataset, nine recasts, nine repetitions, and seven distracters were randomly selected for each NS. The selected episodes were related to the targeted structures and vocabulary items of the task. The template first incorporated a practice session (two distracters and one repetition), followed by video clips of 25 items: nine recasts, nine repetitions, and seven distracters. The order of video clips was randomized using digital video-editing software (Adobe Premiere 6.0). A pause (5-10 s) preceded each video clip, which was then presented three consecutive times. Once the selection and ordering of the video clips was finalized, two videos were prepared for each NS for each of the two conditions in this study (four videos total).

Response-only group. In the video clips for the response-only group, the learner utterances that preceded the NS response on each video clip were digitally erased. The NS response-in the form of a recast, a repetition, or a distracter-was thus seen and heard in isolation from any additional information (linguistic or nonlinguistic) that the NNS utterance might have provided.

Utterance-response group. In the video clips for the utterance-response group, the NNS triggering utterances were not digitally erased. Thus, the learners' initial utterances were heard immediately prior to seeing and hearing the NS's utterance.

These two conditions-one using videotapes including the learners' utterances and the NS responses, and one using videotapes showing only the NS responses-allowed us to address the question of how recasts are recognized as recasts by learners. If learners were equally accurate (or inaccurate) in the rate of recognition, regardless of which videotape they watched, then we might conclude that the contrast between learners' utterances and NS responses is not implicated in learners' perceptions about recasts. However, if there were significant differences in the accuracy of recognition of recasts and repetitions depending on which videotape the learners saw, the contrast between the learner utterance and the response might be a factor in recognition. 


\section{Participants}

Thirty-four learners enrolled in advanced-level classes in English language programs at two universities and two community colleges in the United States participated in this study. Learners from all four settings were highly comparable in terms of biographical details and, as far as possible, the groups were matched in terms of age, years of ESL instruction, and years in an Englishspeaking country. ${ }^{3}$ After balancing the distribution on the basis of institutional setting, group assignment was randomized. The participants, ages 18-39 years $(M=24.6)$, were from a variety of L1 backgrounds and were assigned either to the response-only $(n=17)$ or to the utterance-response $(n=17)$ group. Because the content-based task was not limited to recasts of a specific morphosyntactic form, but rather promoted lexical, phonological, and morphosyntactic recasts in response to whatever errors arose, learners' developmental readiness in relation to the task structures was not assessed. Two English NSs, the same researchers as those featured on the stimulus videotapes, took part in this component of the study as well. The learners were not the same learners featured in the stimulus videotapes.

\section{Procedure}

The experiment was conducted in rooms at the universities/colleges. Each of the sessions was completed within approximately $50 \mathrm{~min}$. Before viewing the stimulus videotapes, the learners were familiarized with the teaching style and discourse moves of the NSs shown on the videotapes; specifically, each learner first completed the same learning and interaction activity that was used to create the videotapes in a dyad with one of the NSs (30 min total). After carrying out the learning activity, NNS viewed the video stimulus that showed the same NS with whom he or she had interacted $(20 \mathrm{~min})$. While the NNS participant viewed the video stimulus, he or she completed a worksheet with written multiple-choice questions about the clips. The options were phrased to convey the key components of the operational definitions of recasts without prompting the learners to look for linguistic contrast. Thus, the NNS participant was instructed to indicate whether he or she thought the NS interlocutor in the video was (a) trying to correct an error by repeating what the NNS had said, (b) repeating a NNS utterance in order to understand him or her, (c) giving instructions, (d) I don't know, or (e) other, with the opportunity to write what he or she thought on a blank line.

A subset of participants in each group (seven per group) also performed think-aloud protocols while completing the worksheets. To demonstrate what was meant by thinking aloud, they were shown a clip of a NNS thinking aloud, and they were asked to say whatever went through their minds while they were watching the video clips and answering the questions. They were instructed that they could think aloud in their native language if they preferred to do so. The think-aloud protocols were audio-recorded. Figure 1 illustrates the experimental procedure in this study. 


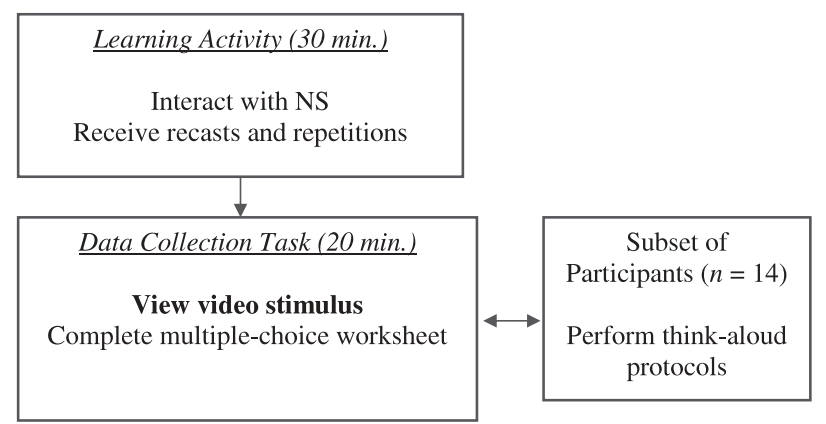

Figure 1. Experimental procedure.

\section{Coding}

Data were coded from two sources: the worksheets and the think-aloud protocols. To address the first research question (Do learners recognize the corrective nature of recasts when the recasts are removed from their immediate discourse context?), scores were generated from the worksheets by summing the number of correctly identified recasts (nine possible). To investigate the relative ambiguity of the different responses, the incorrect answers were separated into recasts misidentified as repetitions and other incorrect answers (i.e., recasts and repetitions misidentified as giving instructions, don't know, other, or no response), and these were also summed (nine possible). Thus, there were nine target items that could have been coded as correctly identified or incorrectly identified; the incorrectly identified answers were further coded as recasts misidentified as repetitions or other incorrect answers. To address the second research question (Do learners identify any nonlinguistic cues when deciding whether utterances are recasts or repetitions?), comments from the think-aloud protocols were coded as either containing explicit reference to nonlinguistic features (e.g., manual or facial gestures), linguistic features (phonological, morphosyntactic, semantic, or discourse level), content, or other. All of the think-aloud protocol data were coded by two raters; two discrepancies were resolved by discussion and input from a third rater. We now turn to a discussion of the results.

\section{RESULTS}

First Research Question: Do Learners Recognize the Corrective Nature of Recasts When the Recasts Are Removed From Their Immediate Discourse Context?

To address the first research question, data were analyzed quantitatively. The descriptive statistics for the average number of times that learners in both 


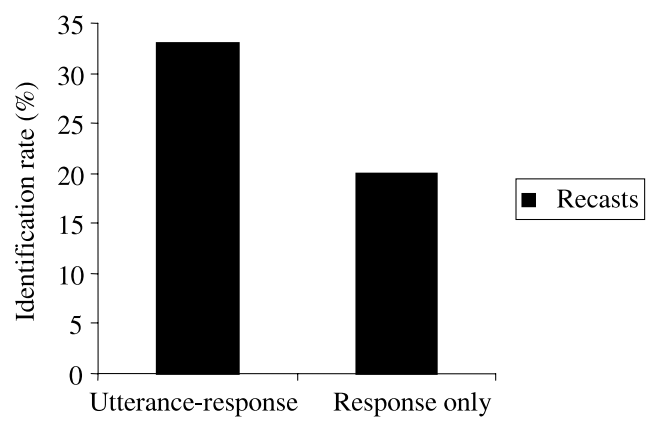

Figure 2. Rates of correct identification of recasts.

groups were able to identify recasts correctly is shown in Table 1. For the response-only group, the mean rate of correct identification of recasts was 1.76 out of 9 (range: $0-5$ ), which was $20 \%$ (30 recasts out of the total 153 provided were recognized). For the utterance-response group, the mean rate of correct identification of recasts was 3.00 out of 9 (range: $1-7$ ), which was $33 \%$ (51 recasts out of the total 153 provided were recognized). ${ }^{4}$ Figure 2 illustrates these results, showing the percentages of correct identifications of recasts for the utterance-response and response-only groups. Group means were compared using $t$-tests (Table 1). The differences between the groups were statistically significant in terms of correct identification of recasts, $t=$ $-2.21, p<.05$. When the learners heard the original utterances (utteranceresponse group), they were more likely to accurately identify recasts as such. ${ }^{5}$

To address the issue of the potential ambiguity of recasts and repetitions, the number of recasts misidentified as repetitions by each of the groups was also compared statistically (Table 1). For the response-only group, the mean rate of misidentification of recasts as repetitions was 4.35 out of 9 (range: $0-8$ ), whereas for the utterance-response group, it was 4.12 out of 9 (range: 1-7). Statistical testing showed no significant differences between the groups,

Table 1. Mean rates of identification of NS recasts and results of $t$-tests

\begin{tabular}{|c|c|c|c|c|c|c|}
\hline \multirow{2}{*}{$\begin{array}{l}\text { Identification } \\
\text { of recasts }\end{array}$} & \multicolumn{2}{|c|}{ Utterance-response $^{\mathrm{a}}$} & \multicolumn{2}{|c|}{ Response only ${ }^{\mathrm{a}}$} & \multirow[b]{2}{*}{$t$} & \multirow[b]{2}{*}{$p$} \\
\hline & $M$ & $S D$ & $M$ & $S D$ & & \\
\hline As recasts & 3.00 & 1.80 & 1.76 & 1.44 & -2.21 & $.03^{*}$ \\
\hline As repetitions & 4.12 & 1.93 & 4.35 & 2.15 & 0.34 & .74 \\
\hline As instructions & 0.00 & 0.00 & 0.76 & 1.35 & 2.34 & $.03^{*}$ \\
\hline Other/Don't know & 1.88 & 1.45 & 2.12 & 1.80 & 0.42 & .68 \\
\hline
\end{tabular}


$t=0.34, p=.74$. In other words, results indicate that even though recasts were more frequently identified as repetitions than recasts by both groups, both groups were equally likely to misidentify them in this way. So, having access to the context of a recast resulted in a group difference in more accurate recognition of recasts, but not in rates of misidentifying recasts as repetitions.

\section{Second Research Question: Do Learners Identify Any Nonlinguistic Cues When Deciding Whether Utterances Are Recasts or Repetitions?}

For the second research question, think-aloud protocols were carried out by a subset of seven randomly selected participants in each group, as explained in the Method section. The subsets of seven learners per group were asked to think aloud as they marked their choices on the worksheet. Data from both groups were coded and compared. Given that the participants in the responseonly group did not hear the original utterances that triggered the NS recasts/ repetitions and did not have the chance to compare the original utterances with the recasts/repetitions, they were expected to focus on nonlinguistic cues more than the utterance-response group, who overheard the NNS utterance; however, as will be discussed later, this prediction was not borne out. ${ }^{6}$

First, the protocol data indicated that learners in both groups were able to articulate the difference between recasts and repetitions. They appeared to understand the corrective nature of recasts, as illustrated in (3) and (4).

(3) Response-only group: Recast identified as recast (Learner A)

I think it's A. Maybe the student says a word, a wrong word or the bad idea, or wrong idea, and he he want to ... to correct this I think it's A.

(4) Utterance-response group: Recast identified as recast (Learner H)

The student said [unintelligible]. What she said, it is not correct, but she [the NS] just said "oh yes," but she pronounce it correctly and she help student to correct an error, uh, correct her pronunciation error.

Learners also seemed to understand that sometimes a repetition is simply a repetition; that is, that there is no corrective element. This can be seen in (5) and (6).

(5) Response-only group: Repetition identified as repetition (Learner B)

Ok. . . umm. . . she repeat uh what a student say uh to understand a student uh B.

(6) Utterance-response group: Repetition identified as repetition (Learner H)

She just repeat what student said and she can motivate student.

Thus, the protocol data show some learners differentiating recasts and repetitions and articulating why they classified utterances as recasts or repetitions. 
Crucially, however, the analysis of the protocol data does not suggest that any of the seven learners in each group who performed think-aloud protocols relied on nonlinguistic cues from the NSs to identify recasts and repetitions. Of 350 opportunities for comments and 252 total comments (with 130 comments for clips pertaining to either a recast or a repetition), there was only one clear reference to the use of nonlinguistic features as a clue to the nature of the utterance. The learner (incorrectly) identified the utterance as a repetition (option B), as shown in (7).

(7) Response-only group: Recast misidentified as repetition (Learner A)

In the finish, this one when he finish the sentence ... he ... he put his face ... for, to, approbation or ok, I understand I think therefore for this face I understand que [that] the option B, is B.

In this episode, a review of the video clip shows that the NS nodded and raised his eyebrows as he provided this recast. After the recast, he nodded again as he raised his eyebrows, frowned, and looked down. No other participants stated in the protocols that they interpreted the NS's nonverbal behavior in this video clip as a sign of approval or understanding, and very few of the participants accurately identified the response as a recast. This was the only case in which a participant made any reference to the facial expressions of the NS.

In six cases, participants explicitly referred to linguistic features when describing why they identified a NS utterance as a recast, as in (4). The example in (8) provides an additional instance in which the participant appears to focus on linguistic code.

(8) Utterance-response group: Recast identified as recast (Learner $\mathrm{H}$ )

She wants to correct the student because the student ... pronunciation is not correct.

In general, most of the think-aloud comments (97 out of 130) focused on meaning, insofar as they were simple statements about what the participants thought the purposes of the responses or exchanges were. When recasts were misidentified as repetitions, participants typically appeared to focus on meaning, as illustrated in (9) and (10).

(9) Response-only group: Recast misidentified as repetition (Learner C) She talk um eh repeat at the student because the student say maybe the waves um ... the waves are um ... [listens again] the waves maybe are ah could be could be dangerous could be ... could be ah ... strong and the people must have uh must have ... warn, warning with, with the waves so.

(10) Utterance-response group: Recast misidentified as repetition (Learner H) I think the instructor want to repeat in order to understand the student. 
Like (9) and (10), most of the participants' comments suggested this focus on the meaning, without any evidence that they were looking for or finding nonlinguistic cues from the NS.

In (11) and (12), the participants specifically referred to their inabilities to distinguish between a recast and a repetition.

(11) Response-only group: Recast misidentified as repetition (Learner A)

I'm not sure $s i$ [if] it's A or B ... I think he is repeating he's repeating the idea what the student say but I'm not sure if it's for correct an error or for understand the student ... oh ... can I say both I don't know ... let me see ... ok I wanna put B again.

(12) Utterance-response group: Recast misidentified as repetition (Learner I)

I don't know if it's like ... the person is correcting the person or understanding the student ... because she's repeating the same thing again ... I don't know which one.

In both examples, the learners clearly understood that the sentence was a repetition of what the NNS said. However, they did not seem to be able to hear or infer the changes made by the researcher, and they explained that they did not know the intent of the researcher. In the end, both participants indicated that they believed the response was a repetition, but in both cases, it was a recast.

\section{DISCUSSION}

The first research question asked whether there was a difference in the learners' abilities to recognize the corrective nature of recasts when the recasts were heard with and without their immediate discourse context. In this study, learners were more accurate at identifying recasts when they heard the learners' original utterances before the recasts. This lends some support to Saxton's (1997) contrast theory of negative evidence; that is, the results of the current study suggest that one key to identifying recasts as such might lie in the immediate context of the interaction rather than in any nonverbal cues that the recaster might provide. As noted earlier, Leeman (2003) discussed three possible sources for the efficacy of recasts in promoting language development-negative feedback, positive evidence, and enhanced salience-and she concluded that it is the enhanced salience of positive evidence in recasts that makes them beneficial. Because the current study was not designed to manipulate the salience of recasts in the same way that Leeman did, the results do not provide direct evidence in support of her conclusions. Nevertheless, they are consistent with her claims in that the immediate context of an exchange might enhance the salience of a recast. Long (in press) pointed out that "if naturally occurring recasts provide added salience to the positive evidence they contain, it is salience accruing from their contingent status in discourse, that is, immediate reformulations..." (p. 35). 
The utterance-response group was able to correctly identify recasts at a higher rate (33\%) than the response-only group (20\%). Still, our data also indicate that access to the learner error did not guarantee that learners could identify the corrective element in recasts. Recasts were more likely to be identified as repetitions than as recasts by both groups. The potential ambiguity of recasts is an important consideration in the evaluation of their pedagogical effectiveness - a point addressed at the beginning of this paper (Long, in press; Lyster, 1998b; Mackey, in press)_and, clearly, the discourse context alone is not always enough for learners to perceive the corrective nature of recasts. There are many potential reasons for this. For example, learners might not be at the correct developmental level to recognize correction (Mackey, 1999) or might experience limitations in aspects of working memory at the time of the recast (Mackey et al., 2002; Philp, 2003). Whatever the reason, the existence of ambiguity is well documented in the literature. Importantly, the presence of ambiguity does not refute the claims of Saxton's contrast theory, which proposes a mechanism by which recasts might promote language development. For recasts to be effective, it is likely necessary only that they be perceived as recasts some, but not all, of the time. ${ }^{7}$ Quality and timing of feedback and perception might be more important than quantity. These questions could be empirically tested.

Also, as discussed in the introduction, it is possible that the type of recast might impact its recognition and effectiveness, as pointed out by Mackey et al. (2000), who found that learners were more likely to recognize the corrective element in phonological or lexical recasts than in morphosyntactic recasts. A post hoc analysis was carried out to ascertain whether there was a relationship between the type of NNS error corrected-phonological, lexical, or morphosyntactic-and the learners' identification patterns. The 18 recasts presented in the videotapes ( 9 per NS interlocutor) were coded by two raters as phonological (4), lexical (5), or morphosyntactic (9) in nature, on the basis of the type of error made by the learner. Two instances in which recasts might have been classified as belonging to more than one category were resolved through discussion and input from a third rater. Examples (13)-(15) illustrate the three types of recasts.

(13) Phonological recast

NNS: A crust.

NS: Yeah, a crest.

(14) Lexical recast

NNS: He broke his surfing board.

NS: He broke his surfboard.

(15) Morphosyntactic recast

NNS: If a storm occur, it's gonna produce a lot of waves.

NS: If a storm occurs, it's gonna produce a lot of waves. 
Table 2 shows the number of correctly and incorrectly identified phonological, lexical, and morphosyntactic recasts for both groups, together with the results of a Mann-Whitney $U$ analysis. There were no significant betweengroup differences in the ability to identify phonological, $U=3.00, p=.15$, lexical, $U=7.50, p=.29$, or morphosyntactic, $U=37.50, p=.79$, recasts. A KruskalWallis test showed that differences in correct identification of different types of recast was significant for the utterance-response group, $\chi^{2}(2)=6.19, p<$ .05 , but not for the response-only group, $\chi^{2}(2)=0.76, p=.69$.

In the current study, both groups viewed more morphosyntactic recasts than lexical or phonological recasts in the content-focused naturalistic interaction. Despite this, learners in the utterance-response group were significantly more accurate in identifying phonological and lexical, but not morphosyntactic, recasts than participants in the response-only group, consistent with Mackey et al.'s (2000) findings. This might be because phonological and lexical recasts tend to be shorter in length than morphosyntactic recasts, which results in a perceptually salient contrast, as argued by Philp (2003). In the current study's data, phonological recasts averaged 3 words, lexical recasts averaged 6 words, and morphosyntactic recasts averaged 10 words; clearly, length and type of recast were related. Another possibility is that phonological and lexical errors are higher in communicative value and more likely to cause communication breakdowns than morphosyntactic ones, which would again increase the saliency of recasts of these errors. In summary, our post hoc analysis uncovered some interesting findings that seem to be worthy of investigation by future research that could balance recast type, length and salience, and communicative value of the target form.

Turning to the second question, which concerned whether learners identify nonlinguistic cues when deciding whether utterances are recasts or repetitions, the analysis of the protocol data shows that, for the most part, the participants in this study did not report observaton of nonlinguistic cues. ${ }^{8}$ Their comments most often focused on the content of the utterances in the clips rather than on any nonlinguistic or linguistic elements of the interaction. Specifically, these results suggest that without the immediate context

Table 2. Frequencies of correct and incorrect identification of different types of recasts and results of the Mann-Whitney $U$-test

\begin{tabular}{|c|c|c|c|c|c|c|c|c|}
\hline \multirow[b]{2}{*}{ Recast type } & \multicolumn{3}{|c|}{ Utterance-response } & \multicolumn{3}{|c|}{ Response only } & \multirow[b]{2}{*}{$U$} & \multirow[b]{2}{*}{$p$} \\
\hline & Correct & Incorrect & Total & Correct & Incorrect & Total & & \\
\hline Phonological & 19 & 14 & 33 & 10 & 29 & 39 & 3.00 & .15 \\
\hline Lexical & 17 & 23 & 40 & 10 & 45 & 55 & 7.50 & .29 \\
\hline Morphosyntactic & 15 & 65 & 80 & 10 & 49 & 59 & 37.50 & .79 \\
\hline Total & 51 & 102 & 153 & 30 & 123 & 153 & & \\
\hline
\end{tabular}


of the utterance that triggers a recast, learners are less able to recognize the corrective nature of the recast. The present study indicates that the opportunity to compare erroneous utterances with the (generally) more targetlike utterances of interlocutors provided by recasts might be one source of any effectiveness attributed to recasts. Although our results suggest that such cognitive comparisons do not occur after the majority of recasts, we believe that it is possible that when they do occur, they are an important first step that might help learners identify what they still need to learn and might, thus, lead to changes in the learners' interlanguage toward more targetlike usage (R. Ellis, 1995; Gass, 2003).

\section{Methodological Innovation}

A great deal of interaction research involves learners carrying out communicative task-based activities similar to the ones used in the current study. The interactions are often audiotaped or videotaped, and instances of interactional modifications (such as recasts) are tallied and analyzed. Most interaction research is based on the hypothesis that interactional modifications result in some kind of change in L2 production or long- or short-term learning (see Mackey \& Gass, this issue). However, as mentioned in the introduction to this paper, the focus on whether interaction leads to L2 learning has led to a new focus in current research, which now typically explores how interaction works to impact learning, with many researchers focusing on learner-internal cognitive processes. New methodological tools are crucial in the investigation of the process-driven questions that are emerging in interaction research. In the current study, we have attempted to move beyond the study of interaction at its surface level to investigate the processes involved in whether and how learners identify recasts as such. Most studies of learners' internal processes use introspective data, and this study follows that trend by using think-aloud protocols to tap into learners' thought processes. However, the current study also uses a unique tool to investigate learners' identification of recasts. The development and administration of edited videotapes, along with an instructional period on the same topic with the same interlocutors, aimed to lower the processing load that accompanies one-on-one interaction. This might offer an advantage for researchers in uncovering how learners interpret NS recasts and repetitions. Moreover, some learners saw and heard recasts isolated from their discourse context, which we hypothesized might free them to use (and comment on) nonverbal or nonlinguistic cues that might accompany recasts. Even though we did not find evidence that learners used such cues, we found significant differences between groups. We interpreted our data as evidence of learners' cognitive comparisons (Baker \& Nelson, 1984; R. Ellis, 1995; Nelson, 1980, 1987) between the initial problematic utterance and the NS's recast. Other explanations for our results are also possible, and we now turn to a discussion of some of these. 


\section{Limitations}

Because this study was carried out in a laboratory setting, the participants were less familiar with their NS interlocutors than most classroom learners are with their teachers. Presumably, classroom learners have time to learn the interactive quirks of their teachers and thus might learn to look for some specific clues that indicate that their teachers are correcting an error. However, a number of studies have shown recasts to be effective in promoting L2 development in the laboratory, even though researchers with whom the participants were unfamiliar provided the recasts. Thus, if such cues are important, we might expect that learners can also identify them in discourses with unfamiliar interlocutors.

It is also possible that the context of the interaction matters in distinguishing recasts from repetitions-not because learners compare the original utterances to the recasts, but because learners need to understand the content of the interaction before they can focus their attention on nonlinguistic cues. In the present study, we attempted to minimize the effects of unfamiliarity with the interaction by showing the participants clips of interaction on the same task that they had just performed themselves; that is, we tried to increase the possibility that the participants would understand the (decontextualized) clips. As discussed further on, the learners in our study were arguably quasi-interactors. Many of the linguistic problems that they saw on the tape replicated ones they had just experienced as they carried out the task. Nevertheless, it is still possible that understanding the structure and content of the task (by way of completing the task themselves) might not have been enough to compensate for the lack of immediate context or the lack of direct engagement or involvement as a participant in the interactions. One putative benefit of recasts is that they address issues that the learner is struggling with at the time of interaction, so that the learner is presumably more attuned to or aware of the corrective content of the recast (Long, 1996, in press). This possibility could be further explored in a replication study in which participants are shown longer stretches of interaction with the recasttriggering utterances removed. Moreover, if the involvement of the learner could be manipulated so that they had struggled with the same linguistic problems controlled for and treated in the video clip, this would raise confidence about the results.

Another issue raised by this study concerns the source of the learner utterance. In most previous recast studies, the focus was on the effect of recasting learners' errors on the learners' interlanguage. In this case, we asked learners to judge recasts and repetitions of other learners' utterances, not their own. This could have hindered or helped the learners' ability to recognize recasts. On the one hand, it is possible that learners are more aware of the content of their own utterances and so better able to judge the intent of a teacher who is recasting or repeating their own error. On the other hand, some researchers have hypothesized that the errors and recasts of other learners are more 
salient because of the lack of communicative pressure (Mackey, 1999). As noted earlier, the research design tried to minimize this effect. All learners participated in the same speech elicitation tasks using the same picture prompts and were provided with mostly identical scripted background information (lesson and instructions). This strategy seemed to work, as evidenced by the fact that many learners made the same vocabulary, conceptual, and communicative errors (e.g., calling a "crest" a "crust," failing to describe a causal connection between pictures). Thus, components of some of the responses that the participants observed in the video were likely to have been experienced firsthand during the immediately preceding half-hour. Therefore, in some sense, the participants in this study were neither observers nor interlocutors, but often chimeras-part observer, part time-delayed quasi-interlocutor. The extent to which their status is a limitation is an empirical question.

It is also arguable that the subset of participants who carried out the thinkaloud protocols ( 7 of 17 participants in each group) impacted the analysis in some way because they performed an additional task in comparison to the other 10 group members. As noted earlier, a careful comparison of data suggests no unusual patterns for these participants and they did not report finding the think-aloud protocols difficult or invasive. Indeed, several learners who were not asked to provide think-aloud protocols did in fact make one or two think-aloud comments to themselves as they filled in the form.

As with all research of this nature, the results of this study might not be applicable beyond the present context (i.e., beyond a laboratory setting with adult learners of English enrolled in college-level language classes). Moreover, the fact that the NS interlocutors were also the researchers might have introduced bias, as they might have unintentionally given clues or unconsciously withheld information that pertained to the corrective element in their recasts.

\section{Future Directions}

The methodology piloted in this study could be applied to address additional questions. For example, a follow-up study could gauge participants' abilities to recognize the (lack of) grammaticality of NNS utterances. In other words, the participants in the utterance-response group might have used a strategy whereby they first identified the grammaticality of the learners' utterances and then, based on this assessment, used reasoning to complete the multiple-choice items; that is, regardless of contrast, participants might have identified responses that followed grammatical utterances as repetitions, whereas those that followed ungrammatical utterances might have been identified as recasts. In this study, the think-aloud protocols do not support this conclusion. However, future research could extend this study by including a group that observes a video stimulus containing only the NNS utterances (the triggers for the recasts or repetitions) and identifies them as grammatical or 
not. It is interesting that the results from the current study indicate that even without access to learners' original errors, and therefore to the contrast in the error-response sequences, learners were able to correctly identify some recasts.

An additional resource that learners might have used to disambiguate recasts and repetitions in this study is (implicit) probabilistic knowledge related to the distributional frequency of such responses in learning/classroom discourse. Probabilistic knowledge about distributional frequencies might underlie many aspects of SLA (N. Ellis, 2005). Performance by learners might reflect a relationship with the distributional frequency of recasts and repetitions within the particular context of the experiment. Learner performance in the responseonly group might reflect this type of knowledge, whereas the context might have permitted learners in the utterance-response group to override such a strategy. The current study cannot address this issue, but further study might shed light on such matters.

The methodology described here could be applied to investigations of other factors that might affect learner recognition of the corrective nature of recasts. As suggested earlier, future research could explore the relationship between different types of recasts and learners' interpretation of them. The post hoc analysis reported here corroborates Mackey et al.'s (2000) finding that morphosyntactic recasts may be more difficult to perceive than other types, such as phonological and lexical recasts; however, length and type of recast were confounded in the current study. Additionally, given that prosody might be effective in resolving syntactic ambiguity (Warren, 1996), whether (and how) learners use prosodic or segmentation cues to disambiguate NS responses could be investigated (Farrar, 1992; Long, in press; Sheen, 2004). Recasts and repetitions that comprise different prosodic qualities and segmentation boundaries could be isolated and compared in a manner similar to that used in this study. The methodology could be further extended to explore participant variables. For example, differences in recognition of recasts as a function of NS gender could be evaluated. Furthermore, individual differences in gender, age, educational attainment, cognitive abilities (e.g., working memory), and personality traits (e.g., openness to correction) could be considered as independent variables.

Finally, the methodology could be used to explore the role that noncorrective repetition plays in language development. The purpose and function of repetitions in interaction do not seem to be the focus in current interaction research, even though there are many claims that learners misinterpret recasts as repetitions in the context of the classroom. In the context investigated here, learners interpreted NS recasts as repetitions more than any other type of utterance. It is possible that repetitions are not ignored by learners, but are seen as immediate mirrors of speech that potentially amplify the learner's utterances and influence development as well. Such research might eventually offer educators concrete guidelines for using recasts and repetitions as pedagogical tools. 


\section{CONCLUSION}

The data in the current study provide some insight into how learners perceive recasts. First, they indicate that with access to learners' original errors and, therefore, to the contrast in the error-response sequences, learners are able to correctly identify recasts better than learners who do not see or hear the original error. Additionally, there is no evidence for the productive use of nonlinguistic cues as a source of disambiguation. This suggests that learners are able to make comparisons between the original utterance and the interlocutor's response. This small-scale but methodologically innovative study suggests that further research that explores the relationship between discourse and cognitive processes is warranted in the ongoing testing of the interaction hypothesis.

\section{NOTES}

1. Brackets indicate clarifications, translations, extralinguistic information, and unintelligible aspects of participant comments.

2. Learners could ask questions or make comments at any point during the instructional phase but rarely did so; they did not need to do so because there were frequent clarification checks.

3. Of 40 original participants, data from 6 were eliminated in order to best match groups in terms of these criteria.

4. Learners' identification of repetitions was also examined. This showed a similar pattern. For the response-only group, the mean rate of correct identification of repetitions was 2.71 out of 9 (range: 0-6), whereas for the utterance-response group, the mean rate of correct identification of repetitions was 4.12 out of 9 (range: 1-8). Statistical testing showed a significant difference between the groups in this respect, $t=2.19, p<.05$. We also examined the rate of misidentification of repetitions as recasts. The mean rate of misidentification of repetitions as recasts for the response only group was 1.88 out of 9 (range: $0-4$ ), whereas it was 2.24 out of 9 (range: $0-7$ ) for the utteranceresponse group. Statistical testing showed that both groups were equally likely to misidentify repetitions as recasts, $t=0.64, p=.53$.

5. A Mann-Whitney U-test was also run because of the small sample size, and it largely confirmed the results. There was a significant difference between the two groups in their ability to identify recasts correctly, $U=87, p<.05$.

6. To allay any concerns that carrying out the think-aloud protocols impacted the participants' identification patterns, data from the 10 participants in each group who did not do the protocols were compared with the data from the 7 who did; similar patterns of results obtained for all 17 learners in each group.

7. It is possible, of course, that learners perceive recasts as positive evidence even if they do not perceive the corrective element, and so might benefit from them.

8. Learners may have varied in the extent to which they watched the video, looked at the worksheet, or focused on something unrelated to the activity.

\section{REFERENCES}

Allwright, D. (1975). Problems in the study of the language teacher's treatment of learner error. In M. Burt \& H. Dulay (Eds.), New directions in second language learning, teaching and bilingual education: On TESOL '75 (pp. 96-109). Washington, DC: TESOL.

Ayoun, D. (2001). The role of negative and positive feedback in the second language acquisition of passé composé and imparfait. Modern Language Journal, 85, 226-243.

Baker, N., \& Nelson, K. (1984). Recasting and related conversational techniques for triggering syntactic advances by young children. First Language, 5, 3-22.

Braidi, S. M. (2002). Reexamining the role of recasts in native-speaker/nonnative-speaker interactions. Language Learning, 52, 1-42. 
Doughty, C., \& Varela, E. (1998). Communicative focus on form. In C. Doughty \& J. Williams (Eds.), Focus on form in classroom second language acquisition (pp. 157-174). New York: Cambridge University Press.

Ellis, N. C. (2005). At the interface: Dynamic interactions of explicit and implicit language knowledge. Studies in Second Language Acquisition, 27, 305-352.

Ellis, R. (1995). Interpretation tasks for grammar teaching. TESOL Quarterly, 29, 87-105.

Fanselow, J. (1977). The treatment of error in oral work. Foreign Language Annals, 10, 583-593.

Farrar, M. J. (1992). Negative evidence and grammatical morpheme acquisition. Developmental Psychology, 28, 90-98.

Gass, S. M. (2003). Input and interaction. In C. Doughty \& M. Long (Eds.), Handbook of second language acquisition (pp. 224-255). Oxford: Blackwell.

Gass, S. M., \& Varonis, E. (1994). Input, interaction, and second language production. Studies in Second Language Acquisition, 16, 283-302.

Han, Z. (2002). A study of the impact of recasts on tense consistency in L2 output. TESOL Quarterly, $36,543-572$.

Havranek, G. (2002). When is corrective feedback most likely to succeed? International Journal of Educational Research, 37, 255-270.

Iwashita, N. (2003). Negative feedback and positive evidence in task-based interaction: Differential effects on L2 development. Studies in Second Language Acquisition, 25, 1-36.

Kessler, C., Lee, L., McCloskey, M., Quinn, M., \& Stack, L. (1996). Making connections: An integrated approach to learning English. Boston: Heinle \& Heinle.

Leeman, J. (2003). Recasts and second language development: Beyond negative evidence. Studies in Second Language Acquisition, 25, 37-63.

Leeman, J. (in press). Feedback. In R. Dekeyser (Ed.), Practice in second language learning: Perspectives from linguistics and psychology. New York: Cambridge University Press.

Loewen, S. (2002). The occurrence and effectiveness of incidental focus on form in meaning-based ESL lessons. Unpublished doctoral thesis, The University of Auckland, New Zealand.

Long, M. H. (1996). The role of the linguistic environment in second language acquisition. In W. C. Ritchie \& T. K. Bhatia (Eds.), Handbook of language acquisition: Vol. 2. Second language acquisition (pp. 413-478). San Diego, CA: Academic Press.

Long, M. H. (Ed.). (in press). Problems in SLA. Mahwah, NJ: Erlbaum.

Long, M. H., Inagaki, S., \& Ortega, L. (1998). The role of implicit negative feedback in SLA: Models and recasts in Japanese and Spanish. Modern Language Journal, 82, 357-371.

Lyster, R. (1998a). Negotiation of form, recasts, and explicit correction in relation to error types and learner repair in immersion classrooms. Language Learning, 48, 183-218.

Lyster, R. (1998b). Recasts, repetition, and ambiguity in L2 classroom discourse. Studies in Second Language Acquisition, 20, 51-81.

Lyster, R. (2004). Differential effects of prompts and recasts in form-focused instruction. Studies in Second Language Acquisition, 26, 399-432.

Lyster, R., \& Ranta, L. (1997). Corrective feedback and learner uptake. Studies in Second Language Acquisition, 19, 37-66.

Mackey, A. (1999). Input, interaction, and second language development: An empirical study of question formation in ESL. Studies in Second Language Acquisition, 21, 557-587.

Mackey, A. (in press). Interaction. In R. DeKeyser (Ed.), Practice in second language learning: Perspectives from linguistics and psychology. New York: Cambridge University Press.

Mackey, A., Gass, S. M., \& McDonough, K. (2000). How do learners perceive interactional feedback? Studies in Second Language Acquisition, 22, 471-497.

Mackey, A., \& Philp, J. (1998). Conversational interaction and second language development: Recasts, responses, and red herrings? Modern Language Journal, 82, 338-356.

Mackey, A., Philp, J., Egi, T., Fujii, A., \& Tatsumi, T. (2002). Individuals differences in working memory, noticing of interactional feedback, and L2 development. In P. Robinson (Ed.), Individual differences and instructed language learning (pp. 181-209). Amsterdam: Benjamins.

Muranoi, H. (2000). Focus on form through interaction enhancement: Integrating formal instruction into a communicative task in EFL classrooms. Language Learning, 50, 617-673.

Nelson, K. (1980). Theories of the child's acquisition of syntax: A look at rare events and at necessary, catalytic, and irrelevant components of mother-child conversation. In V. Teller \& S. White (Eds.), Studies in child language and multiculturalism (pp. 45-68). New York: New York Academy of Sciences.

Nelson, K. (1987). Some observations from the perspective of the rare event cognitive comparison theory of language acquisition. In K. Nelson \& A. Kleeck (Eds.), Children's language (Vol. 6, pp. 289331). Mahwah, NJ: Erlbaum. 
Nicholas, H., Lightbown, P. M., \& Spada, N. (2001). Recasts as feedback to language learners. Language Learning, 51, 719-758.

Ohta, A. S. (2000). Rethinking recasts: A learner-centered examination of corrective feedback in the Japanese language classroom. In J. K. Hall \& L. S. Verplaeste (Eds.), The construction of second and foreign language learning through classroom instruction (pp. 47-71). Mahwah, NJ: Erlbaum.

Oliver, R. (1995). Negative feedback in child NS-NNS conversation. Studies in Second Language Acquisition, 17, 459-481.

Oliver, R. (2000). Age differences in negotiation and feedback in classroom and pairwork. Language Learning, 50, 119-151.

Oliver, R., \& Mackey, A. (2003). Interactional context and feedback in child ESL classrooms. Modern Language Journal, 87, 519-533.

Panova, I., \& Lyster, R. (2002). Patterns of corrective feedback and uptake in an adult ESL classroom. TESOL Quarterly, 36, 573-595.

Philp, J. (2003). Constraints on "noticing the gap": Nonnative speakers' noticing of recasts in NS-NNS interaction. Studies in Second Language Acquisition, 25, 99-126.

Pica, T. (2002). Subject-matter content: How does it assist the interactional and linguistic needs of classroom language learners? Modern Language Journal, 86, 1-19.

Robinson, P. (2001). Task complexity, cognitive resources, and second language syllabus design: A triadic theory of task influences on SLA. In P. Robinson (Ed.), Cognition and second language instruction (pp. 285-317). New York: Cambridge University Press.

Saxton, M. (1997). The contrast theory of negative input. Journal of Child Language, 24, 139-161.

Sheen, Y. (2004). Corrective feedback and learner uptake in communicative classrooms across instructional settings. Language Teaching Research, 8, 263-300.

Skehan, P. (1998). A cognitive approach to language learning. Oxford: Oxford University Press.

Warren, P. (1996). Prosody and parsing: An introduction. Language and Cognitive Processes, 11, 1-16.

\section{APPENDIX}

\section{DESCRIPTION OF TASKS USED IN THIS STUDY}

\section{Task 1, Introduction}

Using an illustration, the NS prompted the NNS to provide vocabulary and descriptions of different phenomena related to waves and their causes. If the NNS mispronounced the vocabulary, provided the wrong lexical item, or made grammatical or other errors, the NS provided recasts. If there were no errors, the NS provided repetitions. Spontaneous discussion was permitted, and additional opportunities for recasts or repetitions that naturally arose were utilized by the NSs as appropriate. Provision of NS responses was dependent on appropriate opportunities to do so in the conversation (cf. Oliver \& Mackey, 2003).

\section{Task 2, Cause and Effect (Factual)}

The NS provided the NNS with five sets of cards that depicted a simple factual causeand-effect relationship (e.g., The boat turned over because of high waves). For example, the first card depicted a fisherman fishing in a stormy sea with high waves, and the second card depicted the fisherman holding on to a lifesaver ring next to a capsized 
boat. Using the first set of cards, the NS first described the series of events using the conditional form that the NNS was expected to produce in the activity. Then, using the same set of cards, the NNS was asked to tell his or her own story. NSs did not provide examples for the subsequent four sets of cards, unless requested by the participant. The NNSs were instructed to first look at both cards and then think about what happened to cause the scene depicted in the second picture. When ready, the NNSs were instructed to tell a story that explained the turn of events on the second card. If the NNS did not use a conditional statement to describe the cause and effect or made any other error, the NS provided a recast. If there were no errors, the NS provided a repetition. Spontaneous discussion was permitted, and additional opportunities for recasts or repetitions that naturally arose were utilized by the NSs as appropriate. Provision of NS responses was dependent on appropriate opportunities to do so in the conversation (cf. Oliver \& Mackey, 2003).

\section{Task 3, Cause and Effect (Counterfactual)}

The NS provided the NNS with three sets of cards that depicted a problem that could be explained using counterfactual cause-and-effect language (e.g., If the friend had surfed in calmer waves, the surfboard would not have broken). For example, the first card depicted a surfer lending his surfboard to a friend, the second depicted the friend surfing in rough water, and the third depicted a broken surfboard. Using the first set of cards, the NS provided an example of how to discuss the pictures to tell a story and used a counterfactual conditional form targeted by the activity. Then, using the same set of cards, the NNS was asked to provide his or her own counterfactual explanation. NS examples were not provided for the subsequent two sets of cards, unless requested by the participant. The NNSs were instructed to first look at all three cards and then to think about the problem depicted in the three pictures. When ready, the NNSs were instructed to explain how the problem might have been prevented. If there were no errors, the NS provided a repetition. Spontaneous discussion was permitted, and additional opportunities for recasts or repetitions that naturally arose were utilized by the NSs as appropriate. Provision of NS responses was dependent on appropriate opportunities in the conversation (cf. Oliver \& Mackey, 2003). 